\title{
OFDR Based Distributed Temperature Sensor Using the Three-Channel Simultaneous Radio-Frequency Lock-In Technique
}

\author{
Ke CHEN, Xinlei ZHOU, Wei PENG, and Qingxu YU* \\ School of Physics and Optoelectronic Technology, Dalian University of Technology, Dalian, 116023, China \\ *Corresponding author: Qingxu YUＥ-mail: yuqx@dlut.edu.cn
}

\begin{abstract}
Weak signal detection for single-mode fiber-optic distributed temperature sensor (DTS) is a key technology to achieve better performance. A hybrid technique combining the incoherent optical frequency domain reflectometry (IOFDR) and the three-channel simultaneous radio-frequency (RF) lock-in amplifier (LIA) is presented to improve the signal-to-noise ratio (SNR) of the measured spontaneous Raman backscattered light. The field programmable gate array (FPGA) based RF-LIA is designed with a novel and simple structure. The measurement frequency range is achieved from $1 \mathrm{kHz}$ to $100 \mathrm{MHz}$. Experimental results show that the backscattered light signal of picowatt level can be detected with high SNR. With a $2.5 \mathrm{~km}$ single-mode fiber, a $1064 \mathrm{~nm}$ laser source, and the measurement time of $500 \mathrm{~s}$, this sensing system can reach a spatial resolution of $0.93 \mathrm{~m}$ and a temperature resolution of about $0.2^{\circ} \mathrm{C}$.
\end{abstract}

Keywords: Distributed temperature sensor, incoherent optical frequency domain reflectometry, radio-frequency lock-in amplifier, simultaneous detection

Citation: Ke CHEN, Xinlei ZHOU, Wei PENG, and Qingxu YU, "OFDR Based Distributed Temperature Sensor Using the Three-Channel Simultaneous Radio-Frequency Lock-In Technique,” Photonic Sensors, 2015, 5(3): 217-223.

\section{Introduction}

The fiber-optic Raman distributed temperature sensor (DTS) can be realized with two different signal detection mechanisms: optical time domain reflectometry (OTDR) and incoherent optical frequency domain reflectometry (IOFDR) [1-3]. Based on the narrow-band signal detection method and the frequency modulated continued wave (FMCW) technology, the IOFDR method has many technical advantages, such as higher signal-to-noise ratio (SNR), lower sample rate, and longer working life of the light source $[4,5]$.

The power of the spontaneous Raman scattering from the optical fiber is about six orders of magnitude weaker than that of the incident light. As a result, low SNR is the main limiting factor for achieving higher spatial resolution, higher temperature resolution, and longer measurement range [6]. This problem is much more serious in the DTS system using single-mode fiber [7, 8]. For an IOFDR-based DTS system, the amplitude of the useful alternating current (AC) signal is dramatically decreased with the modulation frequency increased. The signal power can be attenuated to picowatt level with the modulation frequency up to $50 \mathrm{MHz}$ [8].

By using the electrical heterodyne technique, the received signal can be mixed to a low frequency signal. The digital band-pass filter has been used for

Received version: 6 February 2015 / Revised version: 20 April 2015

(C) The Author(s) 2015.This article is published with open access at Springerlink.com

DOI: $10.1007 / \mathrm{s} 13320-015-0246-\mathrm{z}$

Article type: Regular 
signal processing in IOFDR-based DTS [9], and a temperature resolution of $1.6^{\circ} \mathrm{C}$ was achieved with $3 \mathrm{~m}$ sampling resolution and $13.8 \mathrm{~km}$ single-mode fiber. However, due to the central frequency jitter of the digital filter, the additional noise is increased, which limits the improvements of spatial and temperature resolutions. The radio-frequency (RF) lock-in amplifier (LIA) is suitable for measuring the amplitude and phase of the weak AC signal [10, 11]. The phase-locked loop (PLL) technique used in the LIA can effectively eliminate the frequency jitter caused noise. Meanwhile, for an IOFDR-based DTS system, the signal dynamic range of at least $80 \mathrm{~dB}$ is required. The digital LIA is able to reach a dynamic range up to $110 \mathrm{~dB}$ [12]. By taking advantages of LIA, high SNR improvement and high measurement resolution can possibly be realized.

In this paper, in order to measure the amplitude and the phase shift of the weak Raman backscattered light signal in an IOFDR-based single-mode fiber-optic DTS system, a three-channel simultaneous RF-LIA technique is presented and realized with a novel structure. The SNR improvement of the Raman backscattered light signal is studied by experiments. Furthermore, the spatial and temperature resolutions are measured.

\section{Principles}

\subsection{Principle of the IOFDR-Based DTS}

The IOFDR method uses a continuous wave laser source which is sinusoidal intensity modulated by several stepwise increased frequencies. By using electrical heterodyne technique, the backscattered light signal can be mixed to a low-frequency signal. The AC part of the power of the received light can be expressed as [8]:

$$
\begin{gathered}
\tilde{P}_{i}\left(0, \omega_{m}, t\right)= \\
P_{0} \cdot m \cdot \Re\left(e^{\mathrm{j} \omega_{m} t} \cdot \int_{0}^{L} \chi_{R}(z) \cdot e^{\left(-\left(\alpha_{i}+\alpha_{R}\right) z\right)} \cdot e^{-\mathrm{j} \Omega z} d z\right)
\end{gathered}
$$

where $P_{0}$ is the initial average power of the incident light, $m$ is the modulation depth, $\omega_{m}$ is the modulation frequency, $L$ is the fiber length, $\alpha_{i}$ and $\alpha_{R}$ are the attenuation coefficients of the incident light and the Raman backscattered light, respectively, $\Omega$ is the spatial angular frequency, $\mathfrak{R}$ represents the real part inside the parentheses, and $\chi_{R}(z)$ is the Raman backscattered coefficient at the location of $z$.

With the modulation frequency increased, the amplitude and the phase are measured to obtain the frequency response of the backscattered light signal. Equation (1) indicates that $\chi_{R}(z)$ can be obtained by carrying out an inverse Fourier transform on the measured frequency response, and the profile of the power of backscattered light changing with location can be obtained. Therefore, spatial resolution is achieved by the IOFDR technology.

In the Raman based DTS system, the power of the anti-Stokes component is much more sensitive to temperature, as compared to the Stokes component, because of the Boltzmann distribution of the silica molecular energy level. The DTS technology derives temperature information from the ratio between the powers of the anti-Stokes and Stokes backscattered light [13].

\subsection{Principle of the three-channel simultaneous RF-LIA}

The RF-LIA can be realized by using the electrical heterodyne technique, as shown in Fig.1. The input RF signal is mixed with a local oscillator (LO) whose frequency is higher than the RF signal frequency. With a low-pass filter (LPF), the input signal is converted to an intermediate frequency (IF) signal which can be expressed as

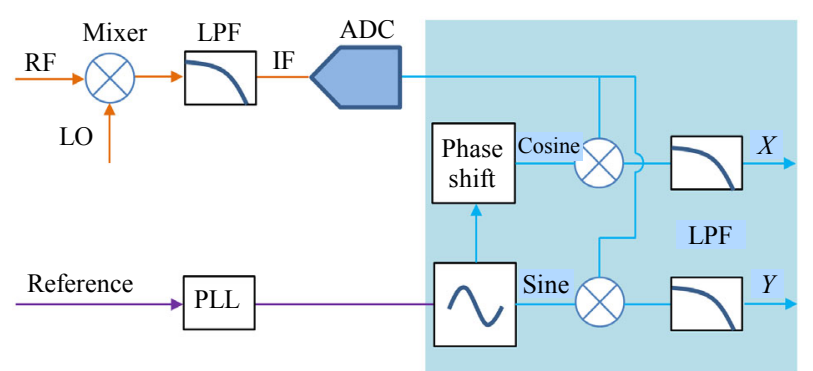

Fig. 1 Schematic diagram of the electrical heterodyne technique based RF lock-in amplifier. 


$$
U_{\mathrm{IF}}=\frac{1}{2} V_{\mathrm{RF}} V_{\mathrm{LO}} \cos \left[\left(\omega_{\mathrm{RF}}-\omega_{\mathrm{LO}}\right) t+\left(\varphi_{\mathrm{RF}}-\varphi_{\mathrm{LO}}\right)\right]
$$

where $V_{\mathrm{RF}}$ and $V_{\mathrm{LO}}$ are the amplitudes of the input $\mathrm{RF}$ signal and the LO signal, $\omega_{\mathrm{RF}}$ and $\omega_{\mathrm{LO}}$ are the frequencies of the RF signal and the LO signal, while $\varphi_{\mathrm{RF}}$ and $\varphi_{\mathrm{LO}}$ are the initial phases of the RF signal and the LO signal.

The frequency of reference signal is equal to the difference frequency between the RF signal and the LO signal, and the reference signal can be expressed as

$$
U_{\mathrm{Ref}}=\cos \left[\left(\omega_{\mathrm{RF}}-\omega_{\mathrm{LO}}\right) t+\varphi_{\mathrm{Ref}}\right]
$$

where $\varphi_{\text {Ref }}$ is the initial phase of the reference signal.

According to the signal detection theory of an orthogonal lock-in amplifier, the measured amplitude and phase of $V_{\mathrm{IF}}$ can be expressed as

$$
\begin{gathered}
\frac{1}{2} V_{\mathrm{RF}} V_{\mathrm{LO}}=2 \sqrt{X_{s}^{2}+Y_{s}^{2}} \\
\varphi_{\mathrm{RF}}-\varphi_{\mathrm{LO}}-\varphi_{\mathrm{Ref}}=\arctan \left(\frac{Y_{s}}{X_{s}}\right)
\end{gathered}
$$

where $X_{\mathrm{s}}$ is the "in-phase" component of the output value, and $Y_{\mathrm{s}}$ is the "quadrature" component.

For an IOFDR based DTS, the initial phase is measured by a reference light channel. Therefore, the measured phase can be expressed as

$$
\varphi_{\mathrm{RL}}-\varphi_{\mathrm{LO}}-\varphi_{\mathrm{Ref}}=\arctan \left(\frac{Y_{r}}{X_{r}}\right)
$$

where $\varphi_{\mathrm{RL}}$ is the initial phase of the reference light, $X_{r}$ is the "in-phase" component of the reference light channel, and $Y_{r}$ is the "quadrature" component.

From (4), the amplitude of the RF signal can be expressed as

$$
V_{\mathrm{RF}}=\frac{4 \sqrt{X_{s}^{2}+Y_{s}^{2}}}{V_{\mathrm{LO}}} .
$$

From (5) and (6), the phase shift of the RF signal can be expressed as

$$
\Delta \varphi=\varphi_{\mathrm{RF}}-\varphi_{\mathrm{RL}}=\arctan \left(\frac{Y_{s}}{X_{s}}\right)-\arctan \left(\frac{Y_{r}}{X_{r}}\right)
$$

With this method, a multi-channel RF-LIA can be developed with a relatively simple structure and few electronic components, compared with traditional methods. Moreover, the amplitude and the phase shift of Raman backscattered light can be measured with a high SNR improvement.

\section{Experimental setup}

\subsection{IOFDR-based DTS setup}

An IOFDR-based DTS system has been developed for well logging, as shown in Fig. 2. Due to harsh environments in oil well, the problem of hydrogen darkening in fiber is serious [14, 15]. Moreover, the caused differential attenuation between Stokes and anti-Stokes channels in the DTS system can lead to a large measurement error. Previous studies have indicated that hydrogen darkening phenomenon can be weakened by using low germanium doped single-mode fiber [16], and the variation of differential attenuation is much smaller by using a laser source whose wavelength is near $1064 \mathrm{~nm}$ [17]. In this scheme, the laser diode, with an emission wavelength of $1064 \mathrm{~nm}$, is modulated by stepwise increased frequencies. A splitter with a coupling ratio of $1: 99$ splits $1 \%$ of the incident light for a reference optical signal which is detected by a photodiode (PD). The laser is launched into the single-mode fiber (SMF) with a length of $2.5 \mathrm{~km}$, and the backscattered lights are detected by an avalanche photodiode (APD) module. Two Raman backscattered lights are filtered out by two wavelength division multiplexers (WDMs). A temperature reference fiber coil with a length of 22 $\mathrm{m}$ is used for calibration. A three-channel simultaneous RF-LIA is designed for laser modulation and signal processing.

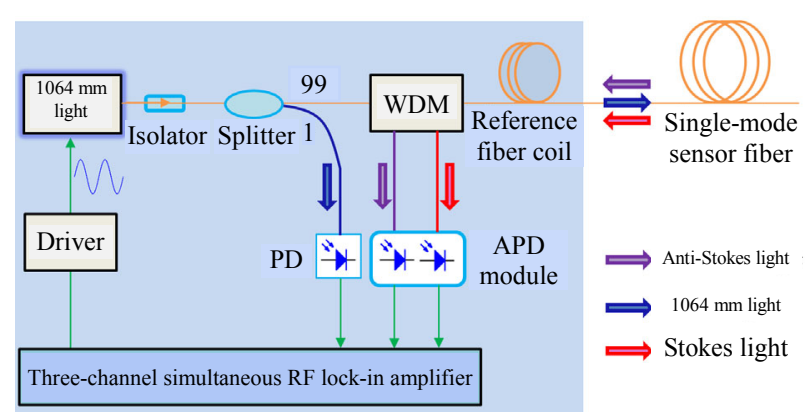

Fig. 2 Setup of the IOFDR-based single-mode fiber-optic DTS system. 


\subsection{Three-channel simultaneous RF-LIA design}

The schematic diagram of the three-channel simultaneous RF-LIA is shown in Fig. 3. The laser modulation signal is supplied by DDS2 which is a direct digital synthesizer (DDS). The laser reference signal, the Stokes signal, and the anti-Stokes signal are mixed with an LO signal whose frequency is $8 \mathrm{kHz}$ higher than the modulation frequency all the time, and the LO signal is supplied by DDS1. The LPF is used to attenuate high frequency component of the output of the mixer. As a result, the three processed signals have the same frequency of $8 \mathrm{kHz}$. The amplitude and the phase of the three ADC converted signals are calculated by the field programmable gate array (FPGA) based digital LIA. Meanwhile, the reference signal input LIA is supplied by DDS3, and its frequency is also $8 \mathrm{kHz}$. A PLL locks the reference signal and multiplies its frequency for ADC sampling clock. In order to minimize the measurement error of the phase, three DDSs are used a same crystal oscillator as their main clock. The size of this RF-LIA is $16 \mathrm{~cm} \times$ $12 \mathrm{~cm} \times 5 \mathrm{~cm}$.

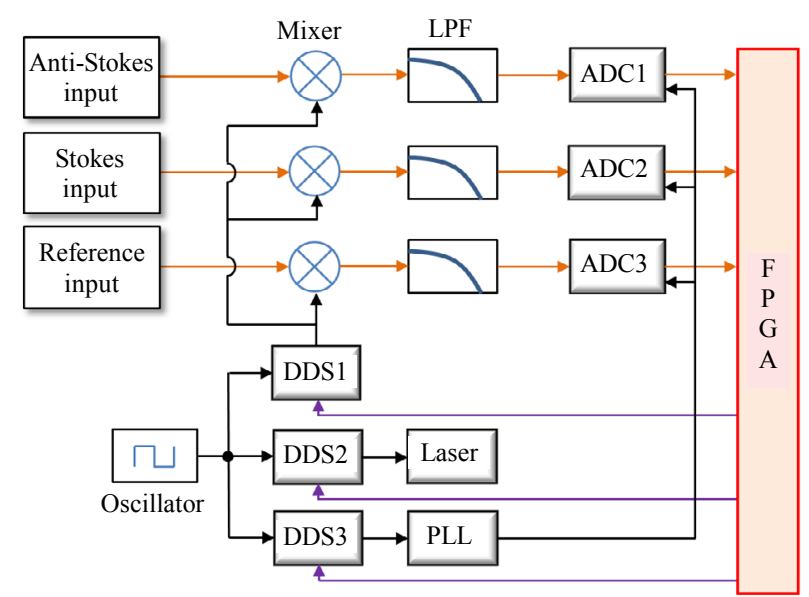

Fig. 3 Schematic diagram of the three-channel simultaneous RF lock-in amplifier.

The sample rate of ADCs is 64 times higher than the reference frequency which is $8 \mathrm{kHz}$. By using this oversampling method, the dynamic range can reach to $114 \mathrm{~dB}$ with a 16-bit ADC. Meanwhile, the trigger signals for sampling are generated by FPGA simultaneously to minimize the error of phase measurement. With the high-speed DDSs, the modulation frequency can sweep from $1 \mathrm{kHz}$ to $100 \mathrm{MHz}$.

With the designed RF-LIA, Raman signals can be processed while data acquiring. After modulation of all frequencies, the demodulation for distributed temperature is carried out by FPGA immediately.

\section{Experimental results and discussion}

\subsection{SNR improvement}

In order to measure the noise spectrum, the measurement frequency was stepwise increased, and the power of the laser diode was not be modulated. Therefore, the Raman backscattered light was a direct-current (DC) signal, and the LIA measured value was the noise near the frequency equal to the reference signal. When the sensor fiber was put into a temperature-controlled chamber which was set to be $30^{\circ} \mathrm{C}$, the average power of the backscattered anti-Stokes light was $24.3 \mathrm{nW}$. Since the noise was relevant to the input optical power [18], the power of the laser diode has been adjusted without modulation until the output power of the backscattered anti-Stokes light to be $24.3 \mathrm{nW}$. Additionally, the standard deviation of the noise of the APD module was measured to be $5.83 \mathrm{mV}$ by an oscilloscope. With the three-channel simultaneous RF-LIA based signal processing unit and a measurement time of $500 \mathrm{~s}$, the measured noise of the APD module over the frequency range of $100 \mathrm{MHz}$ is shown in Fig. 4.

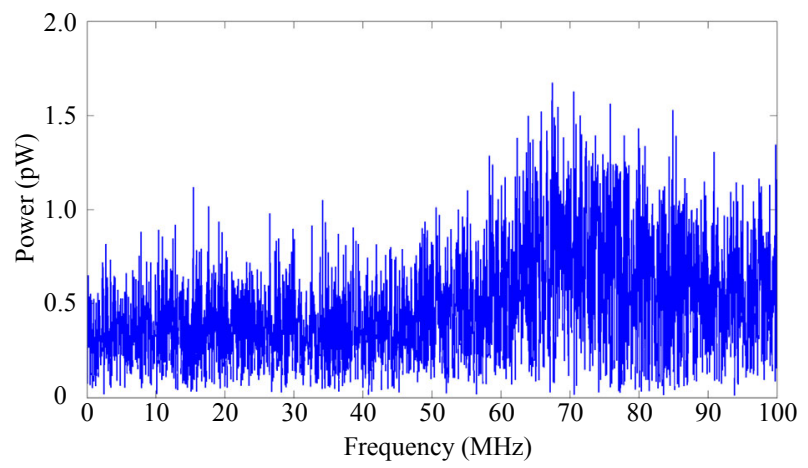

Fig. 4 Measured noise of the APD module over the frequency range of $100 \mathrm{MHz}$. 
Figure 4 shows that the largest noise is near the frequency of $68 \mathrm{MHz}$. Thus an SNR measurement was taken on condition that the laser was modulated with the frequency of $68 \mathrm{MHz}$. The measured anti-Stokes signal value with an integral time of $200 \mathrm{~ms}$ is shown in Fig. 5.

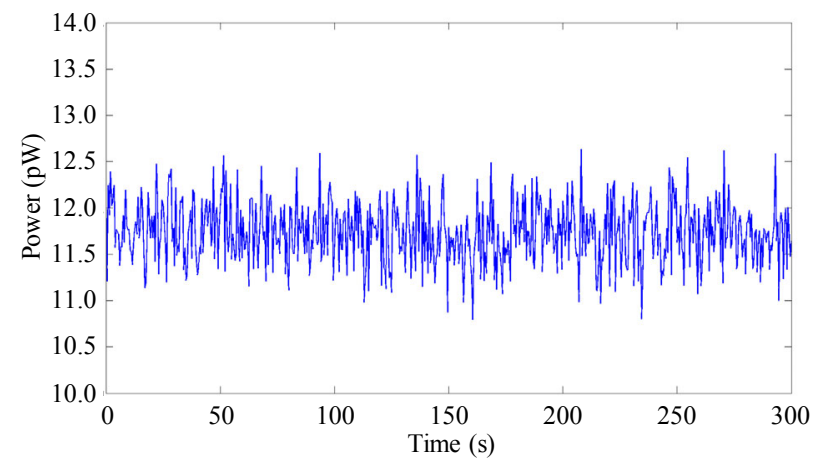

Fig. 5 Measured signal value at the modulation frequency of $68 \mathrm{MHz}$.

From Fig. 5, the root-mean-square (RMS) of the anti-Stokes signal $P_{S}$ is calculated to be $11.7 \mathrm{pW}$, and the standard deviation of the noise $\sigma_{N}$ can be calculated to be $0.33 \mathrm{pW}$. With the responsivity $R$ of the APD module being $1.61 \times 10^{6} \mathrm{~V} / \mathrm{W}$ at $1020 \mathrm{~nm}$, and the gain factor $G$ of the LIA being 11 , the SNR of the LIA output $\mathrm{SNR}_{\mathrm{o}}$ can be calculated as

$$
\mathrm{SNR}_{\mathrm{o}}=10 \cdot \lg \left(\frac{V_{\mathrm{So}}^{2}}{\sigma_{\mathrm{No}}^{2}}\right)=10 \cdot \lg \left(\frac{\left(P_{S} \cdot R \cdot G\right)^{2}}{\left(\sigma_{N} \cdot R \cdot G\right)^{2}}\right)
$$

where $V_{\mathrm{So}}$ is the RMS of the output signal of the LIA, and $\sigma_{\mathrm{No}}$ is the RMS of the output noise. Thus the $\mathrm{SNR}_{\mathrm{o}}$ is calculated to be $30.9 \mathrm{~dB}$.

The input noise $\sigma_{\mathrm{Ni}}$ from the oscilloscope measured value is $5.83 \mathrm{mV}$. The SNR of the LIA input $\mathrm{SNR}_{\mathrm{i}}$ can be calculated as

$$
\mathrm{SNR}_{\mathrm{i}}=10 \cdot \lg \left(\frac{V_{\mathrm{Si}}^{2}}{\sigma_{\mathrm{Ni}}^{2}}\right)=10 \cdot \lg \left(\frac{\left(P_{S} \cdot R\right)^{2}}{\sigma_{\mathrm{Ni}}^{2}}\right)
$$

where $V_{\mathrm{Si}}$ is the RMS of the input signal of the LIA, and $\sigma_{\mathrm{Ni}}$ is the RMS of the input noise. Thus the $\mathrm{SNR}_{\mathrm{i}}$ is calculated to be $-49.8 \mathrm{~dB}$. Therefore, with this RF-LIA, the SNR has been improved from $-49.8 \mathrm{~dB}$ to $30.9 \mathrm{~dB}$. The designed RF-LIA shows a great ability of SNR improvement for weak Raman backscattered signal detection.

\subsection{Frequency response measurement}

With the modulation by stepwise increased frequencies, the Raman backscattered signals were measured by the RF-LIA. To reach a sampling spatial resolution of $0.5 \mathrm{~m}$ and a measurement range of $2.5 \mathrm{~km}$, the maximum modulation frequency was set to be $100 \mathrm{MHz}$, and the frequency interval was set to be $37 \mathrm{kHz}$. When the measurement time has been set to be about $500 \mathrm{~s}$, the amplitude of the measured frequency response of the anti-Stokes channel is shown in Fig. 6(a). The phase shift, which is the difference between the phases of the anti-Stokes signal and the optical reference signal, is shown in Fig. 6(b).

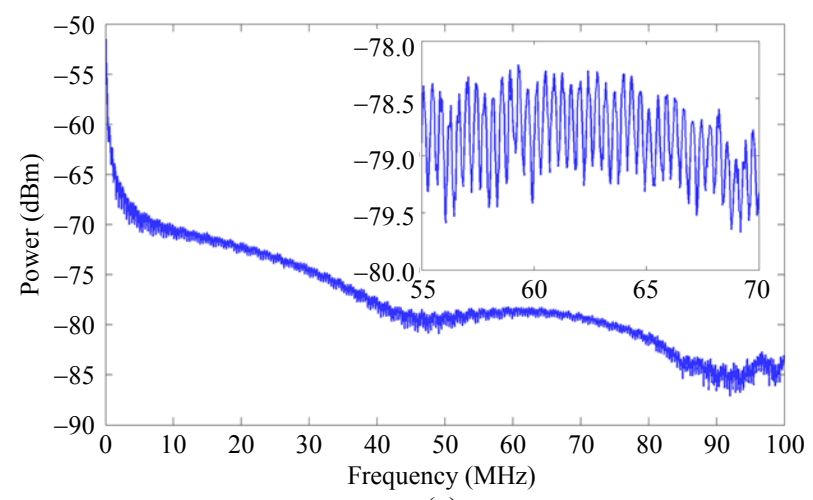

(a)

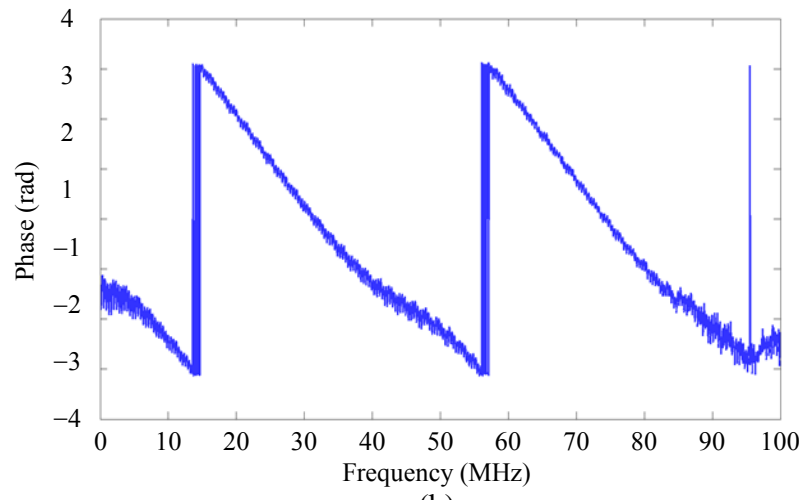

(b)

Fig. 6 Amplitude (a) and the phase shift (b) of the measured frequency response of anti-Stokes channel.

Figure 6(a) shows that the amplitude decreases significantly when the modulation frequency increases. With the modulation frequency up to $50 \mathrm{MHz}$, the power of the AC part of the backscattered light signal could decrease to picowatt level. However, the extremely weak Raman signal 
can still be detected with the RF-LIA. The power changes from near $-50 \mathrm{dBm}$ to $-90 \mathrm{dBm}$, this requires the dynamic range of the signal processing unit exceeding $80 \mathrm{~dB}$. Meanwhile, Fig. 6(b) shows a tendency of decrease in overall, which is caused by a shift of spatial location according to the Fourier transform properties.

\subsection{Distributed temperature measurement}

In order to measure the spatial resolution, the measurement should be carried out at the end of the sensing length where the SNR is the worst, so that the claimed spatial resolution is effective for the entire sensing length [19]. The temperature profile of the tested fiber with a $14 \mathrm{~m}$ fiber coil put into a water bath is shown in Fig. 7. A spatial resolution of $0.93 \mathrm{~m}$ was calculated using the $10 \%$ to $90 \%$ step response corresponding to the rising edge of a temperature change.

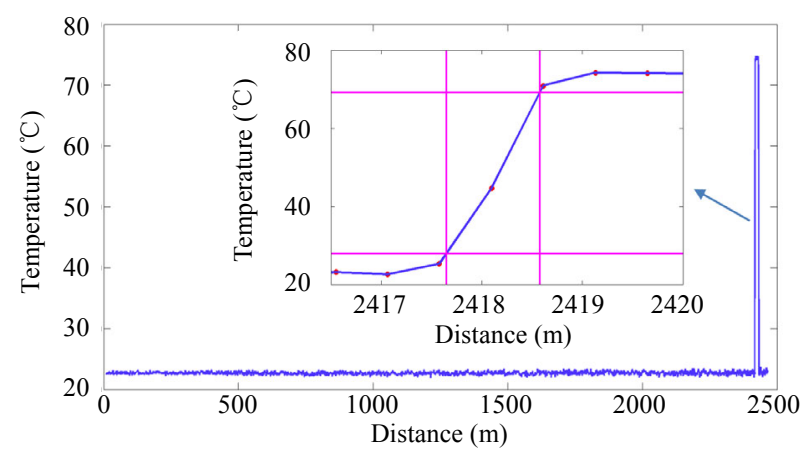

Fig. 7 Measured distributed temperature profile of the $2.5 \mathrm{~km}$ single-mode fiber.

20 distributed temperature profiles were measured with the measurement time of $500 \mathrm{~s}$. The temperature resolution which has been calculated by standard deviation is shown in Fig. 8. The solid line is an exponential fit to the calculated temperature resolution. The fit curve shows that a temperature resolution of about $0.2^{\circ} \mathrm{C}$ has been achieved in the entire measurement range. Due to the increasing attenuation of the propagating light along the fiber, the temperature resolution grows accordingly.

Compared with multi-mode fiber-optic DTS, the Raman scattering cross-section is much smaller in single-mode fiber-optic DTS. Additionally, the responsivity of the detector is much lower near the wavelength of $1064 \mathrm{~nm}$. The SNR is thus reduced with these two factors. Fortunately, the temperature changes slowly in oil well. In order to achieve high temperature resolution for down-hole distributed temperature measurement, long period measurement time is permitted with the single-mode fiber-optic DTS system.

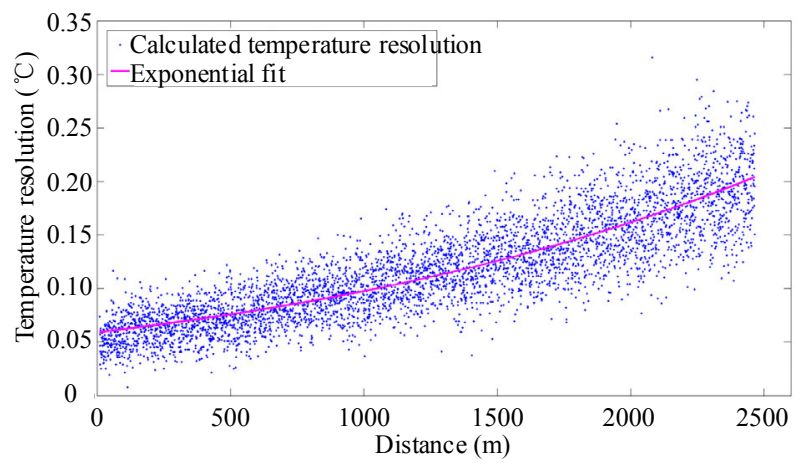

Fig. 8 Temperature resolution along the sensing fiber.

\section{Conclusions}

In conclusion, we have proposed a three-channel simultaneous RF-LIA based signal detection method for an IOFDR-based DTS system. A single-mode fiber-optic DTS has been designed for well logging. In order to detect weak Raman backscattered light, an FPGA based three-channel simultaneous RF-LIA with a novel and simple structure has been designed. At the modulation frequency of $68 \mathrm{MHz}$, the SNR of the anti-Stokes signal was improved from $-49.8 \mathrm{~dB}$ to $30.9 \mathrm{~dB}$. Frequency response of the tested fiber was measured over the frequency range from $36 \mathrm{kHz}$ to $100 \mathrm{MHz}$, and the ability of high sensitivity and large dynamic range for measuring the Raman backscattered light was demonstrated. High spatial resolution and temperature resolution of the distributed temperature profiles have been achieved with high SNR improvement of Raman signal detection. Therefore, the designed three-channel RF-LIA is suitable for frequency response measurement of Raman backscattered light signal in the IOFDR-based DTS system. 


\section{Acknowledgment}

The authors would like to acknowledge the financial supports from the National Nature Science Foundation of China (51277021, 61137005, and 61307105).

Open Access This article is distributed under the terms of the Creative Commons Attribution License which permits any use, distribution, and reproduction in any medium, provided the original author(s) and source are credited.

\section{References}

[1] W. Wang, J. Chang, G. Lv, Z. Wang, Z. Liu, S. Luo, et al., "Wavelength dispersion analysis on fiber-optic Raman distributed temperature sensor system," Photonic Sensors, 2013, 3(3): 256-261.

[2] T. Fukuzawa, H. Shida, K. Oishi, N. Takeuchi, and S. Adachi, "Performance improvements in Raman distributed temperature sensor," Photonic Sensors, 2013, 3(4): 314-319.

[3] G. Bolognini and A. Hartog, "Raman-based fibre sensors: trends and applications," Optical Fiber Technology, 2013, 19(6): 678-688.

[4] M. A. Farahani and T. Gogolla, "Spontaneous Raman scattering in optical fibers with modulated probe light for distributed temperature Raman remote sensing," Journal of Lightwave Technology, 1999, 17(8): 1379-1391.

[5] J. Geng, J. Xu, Y. Li, G. Wei, and C. Guo, "The development of the model and arithmetic for the fully distributed fiber optic sensor based on Raman optical-fiber frequency-domain reflectometry (ROFDR)," Sensors and Actuators A: Physical, 2002, 101(1-2): 132-136.

[6] S. D. Dyer, M. G. Tanner, B. Baek, R. H. Hadfield, and S. W. Nam, "Analysis of a distributed fiber-optic temperature sensor using single-photon detectors," Optics Express, 2012, 20(4): 3456-3466.

[7] L. Meng, M. Jiang, Q. Sui, and D. Feng, "Optical-fiber distributed temperature sensor: design and realization," Optoelectronics letters, 2008, 4(6): 415-418.

[8] E. Karamehmedovic and U. Glombitza, "Fiber optic distributed temperature sensor using incoherent optical frequency domain reflectometry," in Proc. SPIE, vol. 5363, pp. 107-115, 2004.

[9] W. Hill, M. Fromme, J. Kübler, and I. Roda, "Method and apparatus for the spatially resolved measurement of a physical variable," U. S. Patent 8818762, Aug. 26, 2014.

[10] G. Galzerano, E. Bava, R. Ottoboni, and C. Svelto, "Lock-in amplifier up to $530 \mathrm{MHz}$ with phase and amplitude demodulation," in IEEE Instrumentation and Measurement Technology Conference, vol. 2, pp. 1665-1668, 2003.

[11] P. Tian and W. S. Warren, "Ultrafast measurement of two-photon absorption by loss modulation," Optics letters, 2002, 27(18): 1634-1636.

[12] D. B. Gopman, D. Bedau, and A. D. Kent, "A digitally configurable measurement platform using audio cards for high-resolution electronic transport studies," Review of Scientific Instruments, 2012, 83(5): 054701.

[13] Z. Zhang, J. Wang, Y. Li, H. Gong, X. Yu, H. Liu, et al., "Recent progress in distributed optical fiber Raman photon sensors at China Jiliang University," Photonic Sensors, 2012, 2(2): 127-147.

[14] T. Reinsch and J. Henninges, "Temperaturedependent characterization of optical fibres for distributed temperature sensing in hot geothermal wells," Measurement Science and Technology, 2010, 21(9): 094022-1-094022-15.

[15] X. Zhou, Q. Yu, and W. Peng, "Simultaneous measurement of down-hole pressure and distributed temperature with a single fiber," Measurement Science and Technology, 2012, 23(8): 085102-1-085102-7.

[16] W. Hill, J. Kübler, and M. Fromme, "Single-mode distributed temperature sensing using OFDR," in Proc. SPIE, vol. 7653, pp. 765342-765342, 2010.

[17] G. R.Williams, G. Brown, W. Hawthorne, A. H. Hartog, and P. C. Waite, "Distributed temperature sensing (DTS) to characterize the performance of producing oil wells," in Proc. SPIE, vol. 4202, pp. 39-54, 2000.

[18] G. P. Agrawal, Fiber-optic communication systems. New York: Wiley \& Sons, 2002.

[19] X. Bao and L. Chen, "Recent progress in distributed fiber optic sensors," Sensors, 2012, 12(7): 8601-8639. 